\title{
Imaging-Based Assessment of Steatosis, Inflammation and Fibrosis in NAFLD
}

\author{
Timothy Hardy ${ }^{1,2} \cdot$ Stuart McPherson ${ }^{2,3}$
}

Published online: 16 November 2017

(C) The Author(s) 2017. This article is an open access publication

\begin{abstract}
Purpose of Review Non-alcoholic fatty liver disease (NAFLD) is the most common liver disease in the Western world. Invasive liver biopsy remains the gold standard method for the diagnosis and staging of NAFLD. The aim of this review is to summarize recent research regarding imagingbased assessment of NAFLD.

Recent Findings Novel methods such as controlled attenuation parameter (CAP) and magnetic resonance imaging proton-derived fat fraction (MRI-PDFF) appear promising for steatosis assessment and are currently undergoing validation in NAFLD. Fibrosis can be non-invasively assessed by transient elastography (TE), which is currently the best validated test in NAFLD. MR elastography (MRE) appears very sensitive for fibrosis detection. No imaging technique can accurately detect NASH.

Summary TE is inexpensive and relatively widely available and can reliably exclude advanced fibrosis in NAFLD. MRI offers the most promise for steatosis and fibrosis quantification, but further validation of these techniques is needed.
\end{abstract}

This article is part of the Topical Collection on Fatty Liver Disease

Stuart McPherson

stuart.mcpherson@nuth.nhs.uk

Gastroenterology, North Tees University Hospital, Stockton, UK

2 Institute of Cellular Medicine, Faculty of Medical Sciences, Newcastle University, Newcastle Upon Tyne, UK

3 Liver Unit, Newcastle Upon Tyne Hospitals NHS Trust, Freeman Hospital, Level 6, Freeman Road, Newcastle Upon Tyne NE7 7DN, UK
Keywords NAFLD $\cdot$ Steatosis $\cdot$ Fibrosis $\cdot$ Magnetic resonance imaging $\cdot$ Transient elastography $\cdot$ Imaging · Non-invasive test $\cdot$ Ultrasound

\section{Introduction}

Non-alcoholic fatty liver disease (NAFLD) is the leading cause of liver disease worldwide and is estimated to affect $25 \%$ of the global population [1]. NAFLD is strongly associated with obesity, and its prevalence has dramatically increased in the last few decades in parallel with rates of obesity. The histological definition of NAFLD is the presence of triacylglycerol (TAG) droplets in $>5 \%$ of hepatocytes, in the absence of excessive alcohol consumption or the use of steatogenic drugs [2]. Histologically, NAFLD ranges in severity from steatosis alone (frequently know as non-alcoholic fatty liver; NAFL) to steatohepatitis (known as non-alcoholic steatohepatitis; NASH), where steatosis is associated with hepatocellular injury, inflammation and fibrosis. Approximately $40 \%$ of patients with NAFLD will develop progressive fibrosis, which can result in cirrhosis [3, 4]. The incidence of NAFLD-related cirrhosis complications, such as hepatocellular carcinoma and portal hypertension, is increasing, and as a result, NAFLD is projected to be the primary indication for liver transplantation in the US by 2020 [5].

NAFLD has a variable prognosis with the majority of patients having benign disease without associated liverrelated morbidity or mortality. It has been recently determined that the key factor predicting long-term prognosis in patients with NAFLD is the stage of liver fibrosis [6, 7]. Recent studies have shown that individuals with advanced fibrosis (F3-F4) due to NAFLD have a $>3$-fold increased risk of all-cause mortality, compared with a reference 
population $[6,7]$. Therefore, assessment of liver fibrosis is critical in all patients with NAFLD to determine prognosis and appropriate management.

Liver biopsy remains the gold standard test to diagnose and stage NAFLD, but it has many well-documented limitations including the risk of complications, sampling error, inter- and intra-observer variability in reading the histology and the expense of the procedure [8]. Given the large burden of NAFLD worldwide, there is a critical need for simple and accurate non-invasive tests to diagnose hepatic steatosis and stage liver fibrosis. This will help identify individuals at the highest risk of progression so they can be targeted for more aggressive lifestyle interventions or treatments to slow disease progression. There is also an urgent need for accurate quantitative non-invasive tests for steatosis, hepatic inflammation and fibrosis to monitor response to treatment. This is particularly important with the recent development of several new drugs for NAFLD that are going through advanced-phase clinical trials. Currently, there is reliance on liver biopsy to monitor patients in the clinical trials, but this will not be practical once these drugs are widely used.

There have been a number of recent advances in imaging-based assessment of steatosis and fibrosis which are likely to impact on clinical practice. This review aims to highlight some of the recent, important advances in this field and to discuss how these may change clinical practice.

\section{Grading and Staging of Liver Histology as the Reference Standard}

Table 1 shows the histological grading system for NAFLD that is used as the reference standard in most of the recent imaging studies. In the majority, imaging is compared with liver biopsy using semi-quantitative scales for grading steatosis ( $\mathrm{S} 0-3)$ and staging fibrosis $(\mathrm{F} 0-4)$.

Table 1 Histological grading of steatosis and staging fibrosis, according to the NASH CRN [60]

\begin{tabular}{ll}
\hline Steatosis grade $(\mathrm{S} 0-3)$ & Fibrosis $(\mathrm{F} 0-4)$ \\
\hline S0: $0-5 \%$ & F0: None \\
S1: 5-33\% & F1a: Zone 3 mild perisinusoidal \\
& F1b: Zone 3 moderate perisinusoidal \\
& F1c: Periportal/portal only \\
S2: 34-66\% & F2: Perisinusoidal and portal/periportal \\
S3: $>66 \%$ & F3: Bridging fibrosis \\
& F4: Cirrhosis \\
\hline
\end{tabular}

F3-4 = advanced fibrosis
Radiographic Assessment of Steatosis

\section{Ultrasound}

Due to its wide availability and low cost, conventional ultrasound is the most common first line tool for the assessment of hepatic steatosis. A fatty liver appears "bright" compared with surrounding structures due to hepatic TAG deposition, resulting in increased acoustic interfaces. A recent meta-analysis including 2815 patients from 34 studies assessed the diagnostic accuracy of ultrasound compared with histology in patients with NAFLD. Overall, ultrasound had reasonable sensitivity and specificity ( 84.8 and $93.6 \%$, respectively) for detecting $>20$ $30 \%$ steatosis [9]. The sensitivity of ultrasound for detecting milder steatosis $(5-10 \%)$ on liver biopsy was lower, with values as low as $65 \%$ [9]. Ultrasound was also inaccurate in differentiating fatty liver alone, from NASH and liver fibrosis [9]. A clear limitation of ultrasound is its operator dependency, and future studies should include detailed reliability assessments, which are currently lacking. Despite this, ultrasound remains a good first line imaging modality for the assessment of fatty liver. However, as ultrasound is insensitive for mild steatosis, if an individual is strongly suspected of having NAFLD clinically and the ultrasound is reported as normal, then second line imaging modalities should be considered to reduce missed diagnoses.

\section{Controlled Attenuation Parameter}

Controlled attenuation parameter (CAP) is a novel ultrasound technique that measures steatosis simultaneously with liver stiffness during vibration-controlled transient elastography using the FibroScan ${ }^{\mathrm{TM}}$ (Echosens, Paris, France) M or XL Probe. It is known that hepatic steatosis alters the propagation of ultrasound waves through the liver, and CAP measures these physical changes, giving an estimate of steatosis. Results of CAP range from 100 to $400 \mathrm{~dB} / \mathrm{m}$ [10]. The first study describing the efficacy of CAP was published in 2010 and included 115 patients with liver disease from multiple etiologies. In that study, there was a close relationship between CAP and histological assessment of steatosis $\left(r^{2}=0.81\right)$. Moreover, CAP accurately detected $>10$ and $>33 \%$ steatosis with AUROCs of 0.91 and 0.95 , respectively [10]. A subsequent larger study of 153 patients with chronic liver disease and body mass index $\geq 28 \mathrm{~kg} / \mathrm{m}^{2}$ found that the optimum cut-off for detecting $>10 \%$ steatosis was $283 \mathrm{~dB} / \mathrm{m}$ giving a sensitivity and specificity of 76 and $79 \%$, respectively [11]. However, CAP had limited ability to differentiate between specific grades of steatosis, particularly between grades 2 and 3 [11]. 
The largest prospective study assessing CAP in NAFLD included 261 patients and explored the relationship between $\mathrm{CAP}$ and steatosis grade (S0 to $\mathrm{S} 3$ ) and also looked at discordance rates and factors associated with discordance [12]. Overall, CAP values for S0, S1, S2 and $\mathrm{S} 3$ were $264 \pm 45,298 \pm 48,331 \pm 37$ and $336 \pm 31 \mathrm{~dB} / \mathrm{m}$, respectively [12]. Significantly higher $\mathrm{CAP}$ values were seen in patients with $\mathrm{S} 2 / \mathrm{S} 3$ steatosis compared to $\mathrm{S} 1$ (333 versus $285 \mathrm{~dB} / \mathrm{m} ; p<0.001$ ), and the AUROC of CAP to detect $\geq \mathrm{S} 2$ and $\mathrm{S} 3$ was 0.8 and 0.66 , respectively [12]. The optimal CAP cut-off for $\geq \mathrm{S} 2$ disease was $310 \mathrm{~dB} / \mathrm{m}$ [12], which provided a correct assessment of $\mathrm{S} 2 / \mathrm{S} 3$ in $86 \%$ of the cohort [12]. Discordance of at least one grade between CAP and histology was observed in $31 \%$ of the patients.

A meta-analysis including nine studies assessing CAP in patients with chronic liver disease of varying etiologies found overall summary sensitivity and specificity values of 78 and $79 \%$ for $\geq S 1,85$ and $79 \%$ for $\geq S 2$ and 83 and $79 \%$ for S3 [13]. A limitation of this study was that CAP cut-off values were different across the studies and were not validated [13]. In order to address the limitations of this study, a meta-analysis of individual patient data was conducted to establish CAP cut-off values for the diagnosis of steatosis and define values for distinguishing between steatosis grades [14•]. Data from 19/21 eligible studies were available, including 2735 patients ( $>500$ with NAFLD) with chronic liver disease [14•]. In this study, optimal cut-offs were 248 and 268 for $>$ S0 and $>$ S1, respectively [14•]. This study also found that disease etiology may influence the CAP reading, with higher CAP values (by $10 \mathrm{~dB} / \mathrm{m}$ ) for the same grade of steatosis seen in patients with NAFLD compared with individuals with hepatitis $\mathrm{B}$ and $\mathrm{C}[14 \bullet]$.

A major limitation of CAP is its failure rate, which occurred in approximately $20 \%$ of the patients with NAFLD [13]. This was higher than that reported for other etiologies of liver disease where the rate was 7.7\% [15]. Failure of CAP was associated with older age, BMI, presence of metabolic syndrome and female gender, all of which are frequently present in patients with NAFLD [15]. The high failure rate in patients with NAFLD is not surprising as until recently CAP was only available on the FibroScan ${ }^{\mathrm{TM}} \mathrm{M}$ probe. The development of CAP on the XL probe may overcome these limitations [16, 17], but further validation is needed.

Overall, CAP is a relatively simple and inexpensive method for steatosis assessment that is reasonably accurate for the diagnosis of steatosis. When combined with other clinical assessments, it is likely to help clinicians diagnose or exclude steatosis. It also has the advantage that liver stiffness (discussed in the following texts) is measured simultaneously, providing an assessment of fibrosis at the same time. However, CAP appears to have limited ability to differentiate between grades of steatosis and therefore is unlikely to be accurate enough to monitor changes in steatosis with treatment, although this has not been formally assessed.

\section{Magnetic Resonance Imaging-Proton-Derived Fat Fraction (MRI PDFF)}

There are a number of MR techniques that have been described to measure steatosis, including MR spectroscopy (MRS) and conventional MR methods (Dixon in- and out-of-phase imaging) [18]. Until recently, MRS has been the most widely used modality to measure liver fat in large population-based studies and some therapeutic trials [19-24]. However, this technique requires specific expertise and as a result has not been widely used in clinical practice.

More recently, a new method of MRI has been developed called proton density fat fraction (PDFF) which improves on Dixon in- and out-of-phase imaging [25]. Similar to other MR methods, this technique measures steatosis by assessing the fraction of MRI-visible protons bound to fat, divided by all protons bound to fat and water in the liver. The advantage of magnetic resonance imaging-proton-derived fat fraction (MRI-PDFF) over previous methods is that it gives a global assessment of liver fat, whereas techniques, such as MR spectroscopy, measure fat from regions of interest within the liver, which can be associated with sampling variability [26]. Recent cross-sectional studies have shown that PDFF correlates well with histological assessment of steatosis $\left(r^{2}=0.54\right.$, $p<0.001$ and $r^{2}=0.69, p<0.001$, respectively) $[25,27]$ and can accurately distinguish between the presence or absence of hepatic steatosis (AUROC of 0.989) [25]. MRI-PDFF also had good inter-examination accuracy for whole liver assessment $(\mathrm{ICC}=0.999$; SD $<0.24 \%$, range $<0.45 \%$ ) [28]. Furthermore, MRI-PDFF was shown to have better inter- and intra-observer agreement compared with histological steatosis grading $(p<0.001)$ [29].

MRI-PDFF has shown promise in longitudinal studies assessing changes in steatosis with treatment [30-32]. In the FLINT study, a randomized controlled trial (RCT) of obeticholic acid vs. placebo for 72 weeks, MRI-PDFF accurately differentiated steatosis $\mathrm{S} 0-1$ from $\mathrm{S} 2-3$ (AUROC 0.95) and S0-2 from S3 (AUROC 0.96) at baseline [33•]. At $90 \%$ specificity, MRI-PDFF cut-off values were $16.3 \%$ ( $83 \%$ sensitivity) and $21.7 \%$ (84\% sensitivity) for distinguishing histological steatosis $\mathrm{S} 0-1$ from 2 to 3 and S0-2 from S3, respectively [33•]. At week 72 of treatment, $49 \%$ of patients had no change in histological steatosis grade (mean PDFF change $+0.3 \% \pm 6.3 \%), 9 \%$ of patients had increased steatosis 
grade (mean PDFF change $+7.7 \% \pm 6.0 \%$ ) and $42 \%$ of patients had a reduction in steatosis grade (mean PDFF change $-7.4 \% \pm 8.7 \%$ ) [33•]. PDFF change had AUROCs of 0.81 for both a reduction and increase in steatosis grade, respectively [33•]. There was also a good relationship between change in PDFF and change in histological steatosis grade $\left(r^{2}=0.63, p<0.001\right)[33 \bullet]$.

A secondary analysis of the MOZART trial (RCT of ezetimibe versus placebo for 24 weeks in NASH; $n=50$ ) showed a strong correlation between MRIPDFF and total liver volume (TLV) $(r=0.57$, $p<0.0001)$ and MRI-PDFF and total liver fat index (TLFI) $(r=0.94, p<0.0001)$ at week 0 [31]. Patients with grade 3 steatosis on index biopsy had significantly higher PDFF, TLV and TFLI than those with grade 1 steatosis on index biopsy [31]. At week 24, the correlations between the MRI measured indices remained similar, indicating longitudinal reliability [31]. The change in PDFF over the 24-week period also correlated significantly with change in TLV [31]. There was no significant association between the change in TLV and change in histological steatosis at 24 weeks [31]. The authors speculated that within the context of other published studies [32] [19], MRI-PDFF may be more responsive than histological steatosis in detecting incremental hepatic fat change. However, this was a small study, and the authors used grade of steatosis, rather than percentage of steatosis, to assess histology, which may have not been sensitive enough to detect minor histological changes in liver fat [34].

Two recent studies have directly compared MRI-PDFF with CAP for quantifying hepatic steatosis [35••, 36••]. Both studies showed superiority of PDFF over CAP. In the study by Park et al., MRI-PDFF more accurately detected any steatosis than CAP (MRI-PDFF: AUROC 0.99 vs. CAP: AUROC 0.85) and was also more accurate than CAP for identifying specific steatosis grades $(p=0.0091)$ [36••]. Almost identical findings were seen in the other well-conducted study from Japan [35••].

The relationship between MRI-PDFF and histological hepatic steatosis becomes complicated when patients develop advanced liver fibrosis. In the MOZART study, mean PDFF trended downwards between F0-2 and F2-4 $[31,34]$. It is known that MRI may underestimate the amount of fat in hepatocytes when compared with histology in those with more advanced fibrosis because the number of hepatocytes per volume of liver is reduced, leading to an apparent reduction in liver fat [37]. Therefore, it is likely that fibrosis stage will need to be considered when measuring steatosis in patients with chronic liver disease [37]. Concomitant assessment of liver fibrosis and steatosis by MRI may overcome this need [34], as discussed later in this review.
Overall, MRI-PDFF offers an accurate non-invasive assessment of steatosis and may have a role in monitoring a patient's response to treatment. However, MR is expensive, and there is already pressure on MRI scanners for other indications; so, it may not be feasible to use these techniques widely to diagnose and monitor steatosis. However, MRI-PDFF is a useful tool to measure changes in steatosis in clinical trials.

\section{Radiographic Assessment of Liver Stiffness as a Surrogate Biomarker of Fibrosis}

\section{Transient Elastography}

Liver fibrosis can be staged using FibroScan ${ }^{\mathrm{TM}}$ (Echosens, Paris, France), a one-dimensional ultrasound transient elastography (TE), which measures the velocity of a low-frequency $(50 \mathrm{~Hz})$ elastic shear wave, emitted from the ultrasound probe, propagating through the liver [38]. The elastic modulus underpins the measure, i.e. the stiffer the tissue, the faster the shear wave velocity. The liver stiffness (LSM) is measured through a cylindrical volume $1 \mathrm{~cm}$ wide and $4 \mathrm{~cm}$ long, 2-7 $\mathrm{cm}$ below the skin surface. Results are expressed as kilopascals ( $\mathrm{kPa}$ ) using the median of ten valid measurements with a range of 2.5 $75 \mathrm{kPa}$; normal liver has a value of $<5.5 \mathrm{kPa}$ [38]. It is the most commonly used and most validated imaging method for non-invasive assessment of liver fibrosis currently available.

A recent meta-analysis of the use of TE in patients with NAFLD (9 studies, 1047 patients) suggests that TE has excellent diagnostic accuracy for cirrhosis (92\% specificity and $92 \%$ sensitivity), good accuracy for F3 (82\% specificity and $85 \%$ sensitivity), but modest accuracy for F2 (75\% specificity, 79\% sensitivity) [39]. Despite this, TE can rule out cirrhosis with a high NPV ( 90\%) [40]. One of the challenges of using TE is determining the optimum cut-off for diagnosing a specific stage of fibrosis. Generally, a very low LSM is very good at ruling out significant liver fibrosis and a very high LSM indicates cirrhosis with high probability. However, intermediate LSM readings are less accurate, and the choice of a specific cut-off becomes a real balance between specificity and sensitivity. For example, for a diagnosis of cirrhosis, LSM cut-offs between 10.3$17.5 \mathrm{kPa}$ will yield sensitivity of 78 to $100 \%$ and specificity of 82 to $98 \%$ [39]. Therefore, clinicians may use different cut-offs for different stages of fibrosis in different environments depending whether they want to exclude or diagnose a fibrosis stage with high probability. In general, a cut-off of $<8 \mathrm{kPa}$ (or $7.2 \mathrm{kPa}$ for the XL 
probe) reliably excludes advanced fibrosis (F3-4) and a cut-off $>9.6 \mathrm{kPa}$ is suggestive of $\mathrm{F} 3-4$.

A recent population-based, cross-sectional study illustrates the utility of TE as a non-invasive fibrosis marker in detecting clinically relevant liver fibrosis in the community. A total of 3041 participants (aged $\geq 45$ years) from Holland underwent TE with LSM $\geq 8.0 \mathrm{kPa}$, defined as the surrogate marker of clinically relevant liver fibrosis [41•]. Of those with reliable LSM, overall 169 (5.6\%) demonstrated LSM $\geq 8.0 \mathrm{kPa}$, and this increased to $8.4 \%$ in participants with steatosis on ultrasonography and further increased to $17.2 \%$ in those with steatosis and type 2 diabetes [41•]. These findings were consistent with another prospective study from Hong Kong in diabetic patients ( $n=1918)$ which found that $17.7 \%$ of patients had an increased LSM $(\geq 9.6 \mathrm{kPa}$ by $\mathrm{M}$ probe and $\geq 9.6 \mathrm{kPa}$ by $\mathrm{XL}$ probe) [42•]. Of those patients that underwent liver biopsy, $50 \%$ were subsequently diagnosed with advanced fibrosis [42•]. Both these studies indicated the potential to use TE in the community to identify individuals with undiagnosed significant liver disease.

Although the value of TE is clear, it is now recognized that hepatic steatosis has an impact on the accuracy of LSM. This was demonstrated in a recent study of 253 patients with NAFLD, which showed that patients without significant fibrosis (F0-F1) or severe fibrosis (F0-F2) had a higher rate of false-positive LSM results for F3-4 when severe steatosis $(\geq 66 \%)$ was present compared to those with lesser degrees of steatosis (F0-F1 23.6 vs. 14.9\%, F0-F2 33.3 vs. $13.2 \%$, respectively) [43•]. Building on these findings, another study assessed the effect of CAP values on accuracy of LSM in 324 patients with NAFLD using the $M$ probe [44]. CAP values were classified into tertiles (lower 132-298, middle 299-338, higher 339$400 \mathrm{~dB} / \mathrm{m}$ ). Among patients with F0-F2, mean LSM values increased according to CAP tertiles (6.8 versus 8.6 versus 9.4, $p<0.001)$. Moreover AUROCs for the diagnosis of F3-F4 with LSM progressively reduced with increasing CAP tertiles $(0.915,0.830$ and 0.806), and false-positive rates for F3-F4 increased with increasing CAP $(7.2 \%$ in lower versus $16.6 \%$ in middle versus $18.1 \%$ in higher CAP tertiles) [44]. Both studies elegantly define the effect of steatosis on fibrosis prediction, but further studies will need to assess the effect of CAP on LSM values using the XL probe.

A major challenge of using TE in patients with NAFLD is the failure rate (no/insufficient valid measurements) or unreliable results (IQR/LSM $\geq 30 \%$ in patients with LSM $\geq 7.1 \mathrm{kPa}$ ), which was seen in 14.4 and $8.9 \%$ of cases, respectively, in one of the recent well-conducted NAFLD studies [45••]. In the largest series to date $(\geq 13,000$ examinations in 7261 European patients), LSM failure and unreliable results were independently associated with BMI $\geq 30 \mathrm{~kg} / \mathrm{m}^{2}$, age, hypertension, diabetes, operator experience and female gender [46].

As mentioned previously, the XL probe may overcome some of the challenges posed by obese patients. This probe emits a lower central ultrasound frequency, generating a deeper region of interest. The failure rate of the XL probe was significantly lower than that of the M probe $(2 \mathrm{vs}$. $10 \% ; p=0.002$ ), and reliable measurements were obtained in $65 \%$ of obese individuals [47]. A study of patients from France and Hong Kong showed it was possible to obtain valid LSM readings in $91.2 \%$ participants using either the $\mathrm{M}$ or XL probe [48 ${ }^{\bullet}$. However, discordance of $\geq 2$ fibrosis stages between histology and LSM occurred in $11 \%$ of patients using the XL probe, which was 4- to 5-fold more frequent among patients with severe obesity (BMI $\geq 40 \mathrm{~kg} /$ $\mathrm{m}^{2} 32$ vs. $\left.8 \%\right)$ and liver stiffness $>7.0 \mathrm{kPa}(20$ vs. $4 \%)$ [49]. It should be noted that LSM values generated by the $\mathrm{XL}$ probe were lower per stage of fibrosis, and so, distinct cut-off values are required [47, 49]. Finally, LSM may predict long-term prognosis. In a study of 556 patients with NAFLD followed for a median of 6.4 years, individuals with higher LSM had significantly worse overall survival and worse survival free from liver-related and extrahepatic complications [50•].

\section{Acoustic Radiation Force Impulse (ARFI) Imaging}

Acoustic radiation force impulse imaging (ARFI) is a form of elastography that is performed on commercially available B-mode ultrasound machines [51]. ARFI generates short duration acoustic impulses that excite tissues, and the resulting shear wave velocity through the liver is measured giving a liver stiffness measurement. ARFI has the advantage over TE of giving an assessment of the sonographic appearance of the liver and other abdominal structures, as well as giving an estimate of liver fibrosis. A systematic review of seven studies (723 NAFLD patients) showed that ARFI was reasonably accurate for the detection of significant fibrosis ( $\geq$ F2) (AUROC 0.898 ; summary sensitivity and specificity 80.2 and $85.2 \%$, respectively) [52]. However, optimal cut-off values were not assessed in this paper [52]. Much of the work in assessing ARFI as a measure of liver fibrosis has been conducted in patients with viral hepatitis, rather than NAFLD. Therefore, optimum cut-offs for the diagnosis of varying stages of fibrosis in NAFLD need to be validated. There are also different versions of ARFI (such as Siemens Virtual Touch Quantification ${ }^{\mathrm{TM}}$ and Phillips ElastPQ ${ }^{\mathrm{TM}}$ ), which may have different diagnostic accuracies and different diagnostic cutoffs. ElastPQ ${ }^{\mathrm{TM}}$ gives significantly lower ARFI readings than the Virtual Touch quantification ${ }^{\mathrm{TM}}$ [53]; so, these factors need to be considered when using these techniques. 
Another variation of ultrasound elastography is Supersonic Shear Imaging (SSI; Aixplorer, France), which uses an ultrafast ultrasonic scanner [51]. This technique has shown real promise in the assessment of liver fibrosis and performed better than TE in patients with hepatitis $\mathrm{C}$ [54].

\section{Comparison of Liver Stiffness Measurement Techniques}

Only one study compared the diagnostic performance of FibroScan $^{\text {TM }}$ M probe, ARFI (Siemens) and SSI in 291 patients with NAFLD using histology as the reference. Overall, SSI performed best for the detection of significant fibrosis, advanced fibrosis and cirrhosis (AUROCs for SSI, FibroScan ${ }^{\mathrm{TM}}$ and ARFI were 0.86, 0.82 and 0.77 for diagnoses of $\geq F 2 ; 0.89,0.86$ and 0.84 for $\geq \mathrm{F} 3$; and $0.88,0.87$ and 0.84 for $\mathrm{F} 4$; respectively) [45••]. The results are similar to prior individual studies $[39,55]$. It appears that none of the three imaging methods could accurately predict $<$ F2 fibrosis, so further optimization of ultrasound techniques is needed [45••]. Failure rates were lower for ARFI (0.7\%) than for SSI or FibroScan ${ }^{\mathrm{TM}}$ (both $p<0.0001$ ), whereas unreliable results were higher for ARFI (18.2\%) than for SSI or FibroScan ${ }^{\mathrm{TM}}(p=0.0001$ and $p=0.001$, respectively) [45••]. Further trials are needed to confirm the utility of SSI in NAFLD.

\section{Comparison of Liver Stiffness Measurement with Blood Tests}

TE was the best performing test (highest AUROC [0.83], sensitivity [88\%] and NPV [90\%]) for F3-4 when compared with eight blood fibrosis tests (BARD, NAFLD Fibrosis Score, Fibrometer ${ }^{\text {NAFLD }}$, aspartate aminotransferase to platelet ratio index (APRI), FIB4, FibroTest, Hepascore, FibroMeter $^{\mathrm{V} 2 \mathrm{G}}$ ) in a well-conducted study of 452 patients with NAFLD [50•].

\section{Magnetic Resonance Elastography (MRE)}

Magnetic resonance elastography (MRE) is a novel method of elastographic assessment which uses a modified phase-contrast pulse sequence to visualize the propagation of a shear-wave deep into liver tissue, evaluating a large portion of the liver [26]. A recent single-center prospective US study (117 patients) found that MRE very accurately discriminated between F3-4 and F0-2 (AUROC $0.924, p<0.0001)$, but it was less accurate for distinguishing individual fibrosis stages [56••]. A threshold of $\geq 3.63 \mathrm{kPa}$ demonstrated a NPV and PPV of 97 and $68 \%$, respectively, for a diagnosis of F3-4 [56••]. The ability of MRE to discriminate between NASH from
non-NASH was modest (AUROC 0.73) [56••]. Recently, a systematic review evaluated nine studies (232 NAFLD patients) and found that the summary AUROCs for $\geq F 1$, $\geq F 2, \geq F 3$ and F4 were $0.86,0.87,0.90$ and 0.91 , respectively [57].

Given its accuracy, MRE also may offer a good noninvasive tool to monitor changes in liver fibrosis. In a placebo-controlled trial of sitagliptin in NAFLD, MRE was shown to have robust correlation coefficient between baseline and 24 weeks [58]. Longitudinal studies of contemporaneous MRE and liver biopsy are underway, and their results are eagerly awaited.

\section{Comparison of Magnetic Resonance Elastography with Ultrasound-Based Elastography}

Two recent cross-sectional studies have compared the effectiveness of MRE vs. TE for the diagnosis of liver

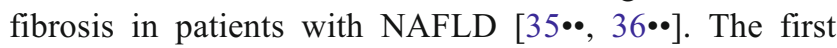
study, from Japan, assessed 142 patients and demonstrated that MRE was superior to TE for the diagnosis of $\geq \mathrm{F} 2$ (AUROCs 0.91 vs. 0.82, respectively; $p=0.001$ ) and F4 (AUROCs 0.97 vs. 0.92; $p=0.049$ ) [35••]. A similar US-based study in 104 patients showed similar findings in a more obese cohort [36••]. In that study, the $\mathrm{M}$ or $\mathrm{XL}$ probe for TE was used where indicated, whereas the Japanese study used the M probe only. Overall, MRE had an AUROC of 0.82 for a diagnosis of any fibrosis (F1-4), which was superior to TE (AUROC 0.67). Using a threshold of $2.65 \mathrm{kPa}, \mathrm{MRE}$ had a sensitivity of $76.5 \%$, specificity of $79.1 \%$, PPV of $81.3 \%$ and NPV of $73.9 \%$ for a diagnosis of any fibrosis [36••]. MRE was also more effective than TE in diagnosing NASH (AUROC 0.70 vs. $0.35 ; p=0.001$ ) $[35 \bullet \bullet, 36 \bullet \bullet$.

Overall, MRE and TE both perform well for a diagnosis of advanced fibrosis and cirrhosis, with MRE being slightly superior. However, MRE outperforms TE for a diagnosis of earlier stages of fibrosis. MRE does not appear to be affected by obesity and ascites, but inflammation and liver iron overload may cause inaccuracies in MRE readings. The major limitation of MRE, when compared with TE, is its cost, and as a result, it is not likely to be widely used for the staging of NAFLD in the community. However, when compared with liver biopsy, the cost of MRE is much more acceptable, and as a result, specialist centers that have the technology available could choose MRE as a second-line investigation for the staging of NAFLD in those where they are considering a liver biopsy. It is also likely that MRE combined with PDFF will continue to be used in clinical trials to monitor response to treatment. 


\section{Multiparametric MRI}

Another novel MRI technique that has been evaluated is multiparametic MRI (liver MultiScan ${ }^{\mathrm{TM}}$, Perspectum Diagnostics, Oxford, England), which assesses hepatic steatosis, fibrosis and iron content. In a study of 79 unselected patients referred for liver biopsy, corrected T1 images (cT1) were significantly different between all fibrosis stages, except between mild and moderate fibrosis [59]. In addition, steatosis measured by rapid spectroscopy correlated strongly with semi-quantitative steatosis scores $\left(r^{2}=0.89, p<0.0001\right)$ [59]. Validation of this technique in larger NAFLD cohorts is needed to accurately assess the clinical utility of this technology.

\section{Conclusions}

With the large burden of disease (mostly undiagnosed) in the community, NAFLD presents a major challenge to healthcare providers. In order to try and reduce the burden of advanced liver disease, it is important to identify individuals with significant fibrosis early so they can be actively managed to reduce fibrosis progression and, where necessary, be screened for cirrhotic complications. In the last 5 years, there has been significant progress in the evaluation of imaging-based techniques to assess both steatosis and fibrosis, with some of the technologies, such as TE, entering routine clinical practice. A proposed algorithm for the detection of steatosis and the assessment of NAFLD severity using imaging is shown in Fig. 1, and a summary of the different imaging modalities for steatosis and fibrosis is shown in Table 2.

TE remains the most widely used imaging technique for fibrosis, and low LSM values can reliably exclude advanced fibrosis, while very high LSM readings indicate cirrhosis with relative certainty. With the development of CAP, steatosis can be assessed simultaneously with fibrosis, with reasonable accuracy. However, TE has modest accuracy for the diagnosis of moderate liver fibrosis, which is a particularly important stage to identify if patients are to receive "disease modifying" treatment.

Of the imaging techniques available, MRI offers the most accurate method for the quantification of steatosis (PDFF) and fibrosis (MRE). MRE is particularly accurate, when compared with $\mathrm{TE}$, in diagnosing milder stages of fibrosis, which is helpful to identify individuals before they have progressed to advanced fibrosis so they can receive treatment. MR also has shown real promise in clinical trials to monitor treatment response, but further

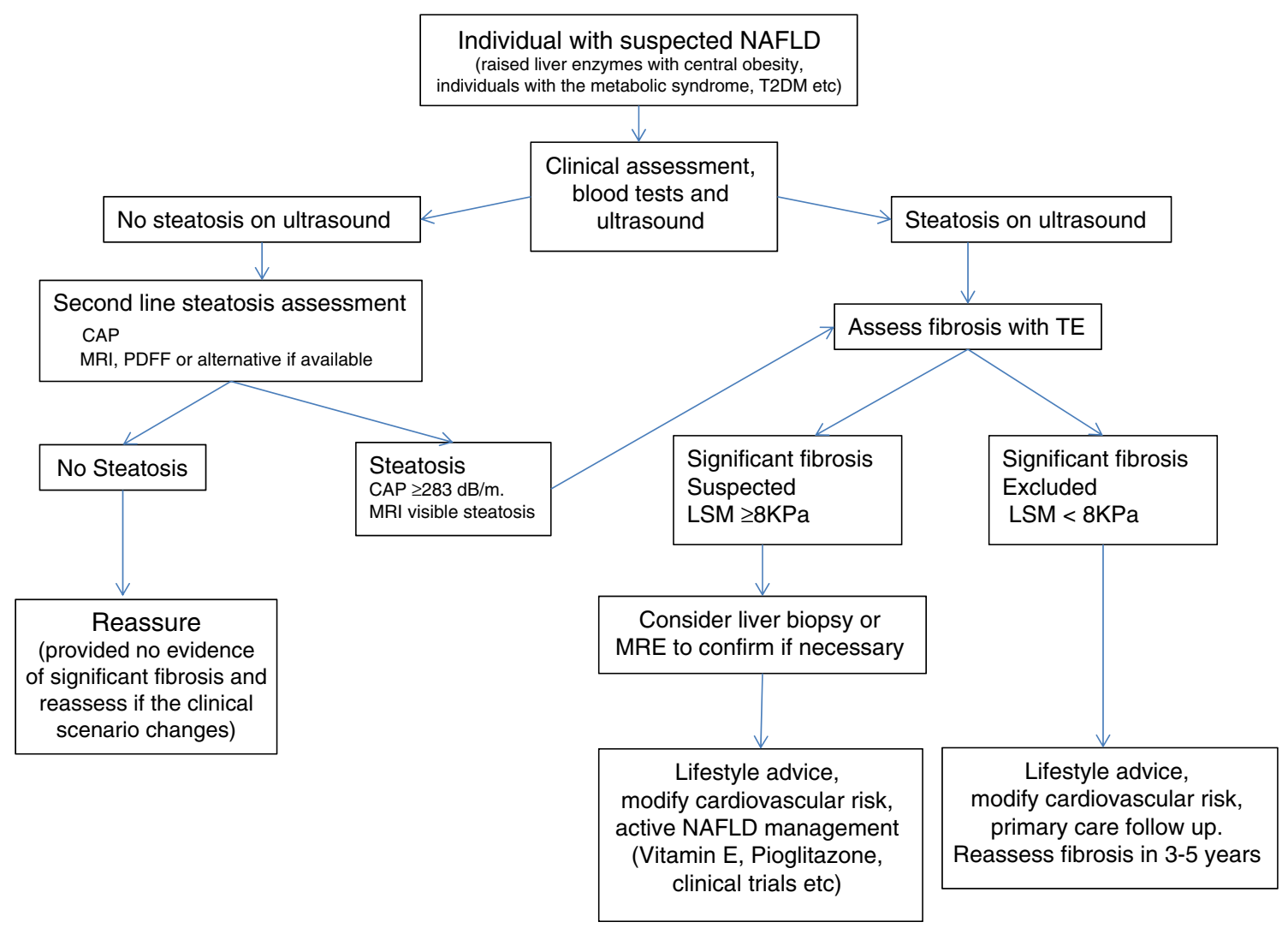

Fig. 1 Proposed diagnostic algorithm for the detection of steatosis and assessment of NAFLD disease severity 
Table 2 Comparison of different modalities in assessing NAFLD disease severity

\begin{tabular}{|c|c|c|c|}
\hline $\begin{array}{l}\text { Imaging } \\
\text { modality }\end{array}$ & Advantages & Disadvantages & Clinical utility \\
\hline US & - Routinely used, low-cost, well-established & $\begin{array}{l}\text { - Poor accuracy at low levels of } \\
\text { hepatic steatosis }<20 \% \\
\text { - Modest reliability } \\
\text { - Cannot discriminate steatosis, } \\
\text { NASH and fibrosis }\end{array}$ & - Good first line test for NAFLD severity assessment \\
\hline CAP & $\begin{array}{l}\text { - Quantitative steatosis assessment } \\
\text { - Can be combined with LSM } \\
\text { - Point of care } \\
\text { - Better accuracy than US }\end{array}$ & $\begin{array}{l}\text { - Cannot discriminate contiguous } \\
\text { steatosis grades } \\
\text { - Limited by obesity, } \\
\text { inflammation and fibrosis }\end{array}$ & - 2nd line steatosis assessment in clinic \\
\hline TE & $\begin{array}{l}\text { - Quantitative fibrosis assessment } \\
\text { - Routinely used in clinic, most validated } \\
\text { - Excellent at excluding cirrhosis } \\
\text { - Provides prognostic information }\end{array}$ & $\begin{array}{l}\text { - Small ROI } \\
\text { - Limited performance in obesity } \\
\text { - False positives in severe } \\
\quad \text { steatosis }\end{array}$ & $\begin{array}{l}\text { - Reliably excludes cirrhosis in a clinic setting, positive results } \\
\text { may still require confirmation (biopsy, MRI) }\end{array}$ \\
\hline ARFI & $\begin{array}{l}\text { - Comparable accuracy to TE for staging } \\
\text { fibrosis and excluding cirrhosis } \\
\text { - Integrated into conventional US machine }\end{array}$ & $\begin{array}{l}\text { - Cannot assess steatosis } \\
\text { - Smaller ROI than TE } \\
\text { - Less well-validated than TE } \\
\text { - TE and SSI outperformed ARFI } \\
\quad \text { for fibrosis } \geq 2 \text { (45) }\end{array}$ & - Exclusion of cirrhosis in a clinic setting \\
\hline SSI & $\begin{array}{l}\text { - Comparable accuracy to TE for staging } \\
\text { fibrosis and excluding cirrhosis } \\
\text { - Integrated into conventional US machine } \\
\text { - Larger ROI than TE and ARFI }\end{array}$ & $\begin{array}{l}\text { - Less well-validated than TE } \\
\text { - Only one study in NAFLD [45] }\end{array}$ & - Needs further prospective study \\
\hline $\begin{array}{l}\mathrm{MRE}+ \\
\quad \mathrm{PDFF}\end{array}$ & $\begin{array}{l}\text { - MRE - excellent diagnostic accuracy for } \\
\text { staging liver fibrosis and cirrhosis } \\
\text { - Steatosis accurately measured with PDFF } \\
\text { - Unaffected by obesity }\end{array}$ & $\begin{array}{l}\text { - More costly than TE } \\
\text { - Requires MRI facility } \\
\text { - Time-consuming } \\
\text { - Failure associated with liver } \\
\text { iron content }\end{array}$ & $\begin{array}{l}\text { - MRE combined with PDFF in clinical trials to monitor response } \\
\text { to treatment, or where liver biopsy is contraindicated }\end{array}$ \\
\hline MMRI & $\begin{array}{l}\text { - No contrast needed } \\
\text { - Samples the entire liver quickly } \\
\text { - Use on existing scanners } \\
\text { - Good correlation with steatosis and fibrosis }\end{array}$ & $\begin{array}{l}\text { - Only single study in various } \\
\text { liver diseases [59] } \\
\text { - Requires MRI facility }\end{array}$ & - Needs further prospective study \\
\hline
\end{tabular}

US, ultrasound; CAP, controlled attenuation parameter; TE, transient elastography; ARFI, acoustic radiation force impulse; SSI, supersonic imaging; MRE, magnetic resonance elastography; MMRI, multiparametic magnetic resonance imaging; LSM, liver stiffness measurement; ROI, region of interest; PDFF, proton density fat fraction; MRI, magnetic resonance imaging; NAFLD, non-alcoholic fatty liver disease; NASH, non-alcoholic steatohepatitis

validation of these techniques is needed. However, critically, the cost-effectiveness, standardization and clinical relevance of MRI evaluation need further study.

\section{Compliance with Ethical Standards}

Conflict of Interest Timothy Hardy and Stuart McPherson declare that they have no potential conflicts of interest.

Human and Animal Rights and Informed Consent This article does not contain any studies with human or animal subjects performed by any of the authors.

Open Access This article is distributed under the terms of the Creative Commons Attribution 4.0 International License (http:// creativecommons.org/licenses/by/4.0/), which permits unrestricted use, distribution, and reproduction in any medium, provided you give appropriate credit to the original author(s) and the source, provide a link to the Creative Commons license, and indicate if changes were made.

\section{References}

Papers of particular interest, published recently, have been highlighted as:

- Of importance

-• Of importance

1. Younossi ZM, Koenig AB, Abdelatif D, Fazel Y, Henry L, Wymer M. Global epidemiology of nonalcoholic fatty liver disease - metaanalytic assessment of prevalence, incidence, and outcomes. Hepatology. 2016;64(1):73-84.

2. Hardy T, Oakley F, Anstee QM, Day CP. Nonalcoholic fatty liver disease: pathogenesis and disease spectrum. Annu Rev Pathol. 2016;11:451-96.

3. Anstee QM, Targher G, Day CP. Progression of NAFLD to diabetes mellitus, cardiovascular disease or cirrhosis. Nature reviews. 2013;10(6):330-44.

4. McPherson S, Hardy T, Henderson E, Burt AD, Day CP, Anstee QM. Evidence of NAFLD progression from steatosis to fibrosingsteatohepatitis using paired biopsies: implications for prognosis and clinical management. J Hepatol. 2015;62(5):1148-55. 
5. Wong RJ, Aguilar M, Cheung R, Perumpail RB, Harrison SA, Younossi ZM, et al. Nonalcoholic steatohepatitis is the second leading etiology of liver disease among adults awaiting liver transplantation in the United States. Gastroenterology. 2015;148(3):547-55.

6. Ekstedt M, Hagstrom H, Nasr P, Fredrikson M, Stal P, Kechagias S, et al. Fibrosis stage is the strongest predictor for disease-specific mortality in NAFLD after up to 33 years of follow-up. Hepatology. 2015;61(5):1547-54.

7. Angulo P, Kleiner DE, Dam-Larsen S, Adams LA, Bjornsson ES, Charatcharoenwitthaya $\mathrm{P}$, et al. Liver fibrosis, but no other histologic features, is associated with long-term outcomes of patients with nonalcoholic fatty liver disease. Gastroenterology. 2015;149(2):389-97. e10

8. Ratziu V, Charlotte F, Heurtier A, Gombert S, Giral P, Bruckert E, et al. Sampling variability of liver biopsy in nonalcoholic fatty liver disease. Gastroenterology. 2005;128(7):1898-906.

9. Hernaez R, Lazo M, Bonekamp S, Kamel I, Brancati FL, Guallar E, et al. Diagnostic accuracy and reliability of ultrasonography for the detection of fatty liver: a meta-analysis. Hepatology. 2011;54(3): 1082-90.

10. Sasso M, Beaugrand M, de Ledinghen V, Douvin C, Marcellin P, Poupon R, et al. Controlled attenuation parameter (CAP): a novel VCTE guided ultrasonic attenuation measurement for the evaluation of hepatic steatosis: preliminary study and validation in a cohort of patients with chronic liver disease from various causes. Ultrasound Med Biol. 2010;36(11):1825-35.

11. Myers RP, Pollett A, Kirsch R, Pomier-Layrargues G, Beaton M, Levstik M, et al. Controlled attenuation parameter (CAP): a noninvasive method for the detection of hepatic steatosis based on transient elastography. Liver International: Official Journal of the International Association for the Study of the Liver. 2012;32(6): 902-10.

12. de Ledinghen V, Wong GL, Vergniol J, Chan HL, Hiriart JB, Chan $\mathrm{AW}$, et al. Controlled attenuation parameter for the diagnosis of steatosis in non-alcoholic fatty liver disease. J Gastroenterol Hepatol. 2016;31(4):848-55.

13. Shi KQ, Tang JZ, Zhu XL, Ying L, Li DW, Gao J, et al. Controlled attenuation parameter for the detection of steatosis severity in chronic liver disease: a meta-analysis of diagnostic accuracy. $\mathrm{J}$ Gastroenterol Hepatol. 2014;29(6):1149-58.

14. Karlas T, Petroff D, Sasso M, Fan JG, Mi YQ, de Ledinghen V, et al. Individual patient data meta-analysis of controlled attenuation parameter (CAP) technology for assessing steatosis. J Hepatol. 2017;66(5):1022-30. Provides individual patient data for meta-analysis to compare cut off values for assessing steatosis.

15. de Ledinghen V, Vergniol J, Capdepont M, Chermak F, Hiriart JB, Cassinotto C, et al. Controlled attenuation parameter (CAP) for the diagnosis of steatosis: a prospective study of 5323 examinations. J Hepatol. 2014;60(5):1026-31.

16. de Ledinghen V, Hiriart JB, Vergniol J, Merrouche W, Bedossa P, Paradis V. Controlled attenuation parameter (CAP) with the XL probe of the Fibroscan $(\mathrm{R})$ : a comparative study with the M probe and liver biopsy. Dig Dis Sci. 2017;

17. Sasso M, Audiere S, Kemgang A, Gaouar F, Corpechot C, Chazouilleres $\mathrm{O}$, et al. Liver steatosis assessed by controlled attenuation parameter (CAP) measured with the XL probe of the FibroScan: a pilot study assessing diagnostic accuracy. Ultrasound Med Biol. 2016;42(1):92-103.

18. Reeder SB, Sirlin CB. Quantification of liver fat with magnetic resonance imaging. Magn Reson Imaging Clin N Am. 2010;18(3):337-57. ix

19. Le TA, Chen J, Changchien C, Peterson MR, Kono Y, Patton H, et al. Effect of colesevelam on liver fat quantified by magnetic resonance in nonalcoholic steatohepatitis: a randomized controlled trial. Hepatology. 2012;56(3):922-32.
20. Heba ER, Desai A, Zand KA, Hamilton G, Wolfson T, Schlein AN, et al. Accuracy and the effect of possible subject-based confounders of magnitude-based MRI for estimating hepatic proton density fat fraction in adults, using MR spectroscopy as reference. J Magn Reson Imaging. 2016;43(2):398-406.

21. Szczepaniak LS, Nurenberg P, Leonard D, Browning JD, Reingold JS, Grundy S, et al. Magnetic resonance spectroscopy to measure hepatic triglyceride content: prevalence of hepatic steatosis in the general population. Am J Physiol Endocrinol Metab. 2005;288(2): E462-8.

22. Bohte AE, van Werven JR, Bipat S, Stoker J. The diagnostic accuracy of US, CT, MRI and $1 \mathrm{H}-\mathrm{MRS}$ for the evaluation of hepatic steatosis compared with liver biopsy: a meta-analysis. Eur Radiol. 2011;21(1):87-97.

23. Houghton D, Thoma C, Hallsworth K, Cassidy S, Hardy T, Burt $\mathrm{AD}$, et al. Exercise reduces liver lipids and visceral adiposity in patients with nonalcoholic steatohepatitis in a randomized controlled trial. Clin Gastroenterol Hepatol. 2017;15(1):96-102. e3

24. Lazo M, Solga SF, Horska A, Bonekamp S, Diehl AM, Brancati FL, et al. Effect of a 12-month intensive lifestyle intervention on hepatic steatosis in adults with type 2 diabetes. Diabetes Care. 2010;33(10):2156-63.

25. Tang A, Tan J, Sun M, Hamilton G, Bydder M, Wolfson T, et al. Nonalcoholic fatty liver disease: MR imaging of liver proton density fat fraction to assess hepatic steatosis. Radiology. 2013;267(2): 422-31.

26. Dulai PS, Sirlin CB, Loomba RMRI. MRE for non-invasive quantitative assessment of hepatic steatosis and fibrosis in NAFLD and NASH: clinical trials to clinical practice. J Hepatol. 2016;65(5): 1006-16.

27. Permutt Z, Le TA, Peterson MR, Seki E, Brenner DA, Sirlin C, et al. Correlation between liver histology and novel magnetic resonance imaging in adult patients with non-alcoholic fatty liver diseaseMRI accurately quantifies hepatic steatosis in NAFLD. Aliment Pharmacol Ther. 2012;36(1):22-9.

28. Negrete LM, Middleton MS, Clark L, Wolfson T, Gamst AC, Lam $\mathrm{J}$, et al. Inter-examination precision of magnitude-based MRI for estimation of segmental hepatic proton density fat fraction in obese subjects. J Magn Reson Imaging. 2014;39(5):1265-71.

29. Bannas P, Kramer H, Hernando D, Agni R, Cunningham AM, Mandal R, et al. Quantitative magnetic resonance imaging of hepatic steatosis: validation in ex vivo human livers. Hepatology. 2015;62(5):1444-55.

30. Patel J, Bettencourt R, Cui J, Salotti J, Hooker J, Bhatt A, et al. Association of noninvasive quantitative decline in liver fat content on MRI with histologic response in nonalcoholic steatohepatitis. Therap Adv Gastroenterol. 2016;9(5):692-701.

31. Lin SC, Heba E, Bettencourt R, Lin GY, Valasek MA, Lunde O, et al. Assessment of treatment response in non-alcoholic steatohepatitis using advanced magnetic resonance imaging. Aliment Pharmacol Ther. 2017;45(6):844-54.

32. Noureddin M, Lam J, Peterson MR, Middleton M, Hamilton G, Le TA, et al. Utility of magnetic resonance imaging versus histology for quantifying changes in liver fat in nonalcoholic fatty liver disease trials. Hepatology. 2013;58(6):1930-40.

33. Middleton MS, Heba ER, Hooker CA, Bashir MR, Fowler KJ, Sandrasegaran K, et al. Agreement between magnetic resonance imaging proton density fat fraction measurements and pathologist-assigned steatosis grades of liver biopsies from adults with nonalcoholic steatohepatitis. Gastroenterology. 2017.Paper uses data from the FLINT study and shows the utility of MRI-PDFF for longitudinal assessment of steatosis.

34. Mansour D, McPherson S. Editorial: further evidence for the use of advanced magnetic resonance imaging techniques to monitor NAFLD. Aliment Pharmacol Ther. 2017;45(9):1269-70. 
35.• Imajo K, Kessoku T, Honda Y, Tomeno W, Ogawa Y, Mawatari H, et al. Magnetic resonance imaging more accurately classifies steatosis and fibrosis in patients with nonalcoholic fatty liver disease than transient elastography. Gastroenterology. 2016;150(3): 626-37. e7. Well-conducted study comparing for the first time MR vs. US- based imaging methods for detection of steatosis and fibrosis in NAFLD patients.

36.• Park CC, Nguyen P, Hernandez C, Bettencourt R, Ramirez K, Fortney L, et al. Magnetic resonance elastography vs transient elastography in detection of fibrosis and noninvasive measurement of steatosis in patients with biopsy-proven nonalcoholic fatty liver disease. Gastroenterology. 2017;152(3):598-607. e2. Well-conducted, US-based study comparing MR vs. US- based methods for steatosis and fibrosis detection.

37. McPherson S, Jonsson JR, Cowin GJ, O'Rourke P, Clouston AD, Volp A, et al. Magnetic resonance imaging and spectroscopy accurately estimate the severity of steatosis provided the stage of fibrosis is considered. J Hepatol. 2009;51(2):389-97.

38. Sandrin L, Fourquet B, Hasquenoph JM, Yon S, Fournier C, Mal F, et al. Transient elastography: a new noninvasive method for assessment of hepatic fibrosis. Ultrasound Med Biol. 2003;29(12):170513.

39. Kwok R, Tse YK, Wong GL, Ha Y, Lee AU, Ngu MC, et al. Systematic review with meta-analysis: non-invasive assessment of non-alcoholic fatty liver disease - the role of transient elastography and plasma cytokeratin-18 fragments. Aliment Pharmacol Ther. 2014;39(3):254-69.

40. European Association for Study of L, Asociacion Latinoamericana para el Estudio del H. EASL-ALEH clinical practice guidelines: non-invasive tests for evaluation of liver disease severity and prognosis. J Hepatol. 2015;63(1):237-64.

41. Koehler EM, Plompen EP, Schouten JN, Hansen BE, Darwish Murad S, Taimr P, et al. Presence of diabetes mellitus and steatosis is associated with liver stiffness in a general population: the Rotterdam study. Hepatology. 2016;63(1):138-47. Demonstrates the clinical utility of TE in a population-based setting.

42. Kwok R, Choi KC, Wong GL, Zhang Y, Chan HL, Luk AO, et al. Screening diabetic patients for non-alcoholic fatty liver disease with controlled attenuation parameter and liver stiffness measurements: a prospective cohort study. Gut. 2016;65(8):1359-68. Elegant study demonstrating the utility of TE in screening diabetic patients for liver disease.

43. Petta S, Maida M, Macaluso FS, Di Marco V, Camma C, Cabibi D, et al. The severity of steatosis influences liver stiffness measurement in patients with nonalcoholic fatty liver disease. Hepatology. 2015;62(4):1101-10. Excellent study demonstrating that inaccurate TE measurements may be mitigated by CAP.

44. Petta S, Wong VW, Camma C, Hiriart JB, Wong GL, Marra F, et al. Improved noninvasive prediction of liver fibrosis by liver stiffness measurement in patients with nonalcoholic fatty liver disease accounting for controlled attenuation parameter values. Hepatology. 2017;65(4):1145-55.

45.• Cassinotto C, Boursier J, de Ledinghen V, Lebigot J, Lapuyade B, Cales P, et al. Liver stiffness in nonalcoholic fatty liver disease: a comparison of supersonic shear imaging, FibroScan, and ARFI with liver biopsy. Hepatology. 2016;63(6):1817-27. Landmark study comparing the clinical utility of several liver stiffness modalities for the detection of fibrosis in NAFLD patients.

46. Castera L, Foucher J, Bernard PH, Carvalho F, Allaix D, Merrouche $\mathrm{W}$, et al. Pitfalls of liver stiffness measurement: a 5-year prospective study of 13,369 examinations. Hepatology. 2010;51(3):828-35.
47. Wong VW, Vergniol J, Wong GL, Foucher J, Chan AW, Chermak F, et al. Liver stiffness measurement using XL probe in patients with nonalcoholic fatty liver disease. Am J Gastroenterol. 2012;107(12): $1862-71$.

48. de Ledinghen V, Wong VW, Vergniol J, Wong GL, Foucher J, Chu $\mathrm{SH}$, et al. Diagnosis of liver fibrosis and cirrhosis using liver stiffness measurement: comparison between $\mathrm{M}$ and XL probe of FibroScan(R). J Hepatol. 2012;56(4):833-9. Well conducted study showing unreliable results using the $M$ probe are mitigated by use of XL probe.

49. Myers RP, Pomier-Layrargues G, Kirsch R, Pollett A, Beaton M, Levstik M, et al. Discordance in fibrosis staging between liver biopsy and transient elastography using the FibroScan XL probe. J Hepatol. 2012;56(3):564-70.

50. Boursier J, Vergniol J, Guillet A, Hiriart JB, Lannes A, Le Bail B, et al. Diagnostic accuracy and prognostic significance of blood fibrosis tests and liver stiffness measurement by FibroScan in non-alcoholic fatty liver disease. J Hepatol. 2016;65(3):570-8. First study to demonstrate the prognostic relevance of TE, and compare TE with well established blood tests for fibrosis detection.

51. Sporea I, Gilja OH, Bota S, Sirli R, Popescu A. Liver elastography — an update. Med Ultrason. 2013;15(4):304-14.

52. Liu H, Fu J, Hong R, Liu L, Li F. Acoustic radiation force impulse elastography for the non-invasive evaluation of hepatic fibrosis in non-alcoholic fatty liver disease patients: a systematic review \& meta-analysis. PLoS One. 2015;10(7):e0127782.

53. Sporea I, Bota S, Gradinaru-Tascau O, Sirli R, Popescu A. Comparative study between two point shear wave elastographic techniques: acoustic radiation force impulse (ARFI) elastography and ElastPQ. Med Ultrason. 2014;16(4):309-14.

54. Bavu E, Gennisson JL, Couade M, Bercoff J, Mallet V, Fink M, et al. Noninvasive in vivo liver fibrosis evaluation using supersonic shear imaging: a clinical study on 113 hepatitis $\mathrm{C}$ virus patients. Ultrasound Med Biol. 2011;37(9):1361-73.

55. Cui J, Heba E, Hernandez C, Haufe W, Hooker J, Andre MP, et al. Magnetic resonance elastography is superior to acoustic radiation force impulse for the diagnosis of fibrosis in patients with biopsyproven nonalcoholic fatty liver disease: a prospective study. Hepatology. 2016;63(2):453-61.

56.• Loomba R, Wolfson T, Ang B, Hooker J, Behling C, Peterson M, et al. Magnetic resonance elastography predicts advanced fibrosis in patients with nonalcoholic fatty liver disease: a prospective study. Hepatology. 2014;60(6):1920-8. Landmark study demonstrating the efficacy of MRE for detecting fibrosis in NAFLD patients.

57. Singh S, Venkatesh SK, Loomba R, Wang Z, Sirlin C, Chen J, et al. Magnetic resonance elastography for staging liver fibrosis in nonalcoholic fatty liver disease: a diagnostic accuracy systematic review and individual participant data pooled analysis. Eur Radiol. 2016;26(5):1431-40.

58. Cui J, Philo L, Nguyen P, Hofflich H, Hernandez C, Bettencourt R, et al. Sitagliptin vs. placebo for non-alcoholic fatty liver disease: a randomized controlled trial. J Hepatol. 2016;65(2):369-76.

59. Banerjee R, Pavlides M, Tunnicliffe EM, Piechnik SK, Sarania N, Philips R, et al. Multiparametric magnetic resonance for the noninvasive diagnosis of liver disease. J Hepatol. 2014;60(1):69-77.

60. Kleiner DE, Brunt EM, Van Natta M, Behling C, Contos MJ, Cummings OW, et al. Design and validation of a histological scoring system for nonalcoholic fatty liver disease. Hepatology. 2005;41(6):1313-21. 